\title{
Sound Source Characterisation and Transfer Path Analysis with Time Histories
}

\author{
Nicklas Frenne and Örjan Johansson ${ }^{\dagger}$ \\ Luleå University of Technology, Dept. of Human Work Science, Div. of Sound and Vibration, S-971 87 Luleå, Sweden
}

(Received 2 April 2002; revised 6 January 2003; accepted 6 August 2003)

\begin{abstract}
The article describes a method to separate time histories of partial sound sources. The goal is to develop a noise control engineering tool for use in sound quality improvement applications. Contributions from partial sound sources are identified. The partial sound sources may be ranked for the purpose of creating a better mixture of sound in selected listening positions. The strategy is to reproduce time histories of sources of importance. The method described includes experimental and calculation parts. The experimental part consists of the recording of sound pressure time signals, reciprocal measurement of frequency response functions, and source strength estimation of partial sound sources. The calculation part comprises calculation of the cross-spectral matrix of source strength, calculation of filters, and filtered sound pressure recording to obtain time signals of the individual sources. Usually the contribution from partial sources is impossible to record directly. In this laboratory experiment, such control was possible. The laboratory experiment shows that the method described makes it possible to produce informative separation of time histories of partial sound sources. The effects of the errors in the calculated time histories are audible but not pronounced.
\end{abstract}

${ }^{\dagger}$ Member of the International Institute of Acoustics and Vibration (IIAV)

\section{INTRODUCTION}

Situations where the surrounding sound field is generated by multiple sources occur frequently in many work environments, environments are found including heavy vehicles and/or control rooms. Good sound in such environments is generally described as an appropriate balance between background noise and adequate acoustical feedback from the controlled processes. To achieve this, noise control engineers require a method to separate the contribution from different sound sources. When the contribution from different sources is determined, a good balance among these sources can be proposed through listening tests. After that, the optimisation of necessary control actions on sources that degrade the sound is possible.

Several methods for source separation have been described. One example is transfer path analysis. ${ }^{1-3}$ However, these methods of transfer path analysis have not focused on retrieving the time histories of sound signals. The objective of this study is to develop and validate a method that retrieves sound signals of piston sound sources to give a foundation for future studies of complex sound sources.

The method is partitioned into six steps: 1) recording of sound pressure time signals with a set of microphones; 2) reciprocal measurement ${ }^{4}$ of frequency response functions that describe how the sound is altered between the microphones and a limited number of defined sound sources; 3) source strength estimation of sound sources; 4) calculation of crossspectral matrix of source strength $\left.\boldsymbol{S}_{q q} ; 5\right)$ calculation of filters; and 6) filtered sound pressure recordings.

The method is tested and validated in a laboratory experiment in which sounds from two loudspeakers are separated. The result is displayed with spectra from separated signals and tables with deviations for different frequency regions.
The result shows that it is possible to separate time signals of sound sources with an inverse approach. ${ }^{5}$

A plausible application of a generalised method is the separation of sound from different sources that contribute to the sound in a control room in a factory or a truck cabin. Receiver positions, such as the ears of an artificial head, can be chosen. The result can then be assessed with auxiliary equipment associated with binaural technology. ${ }^{6}$

\section{THEORY}

\subsection{Source Model}

Three different source models are described. The models are used to discretise the sources into formats that are convenient and useful. The choice of model depends on the frequency region of interest, size of object and correlation pattern of the sources.

\section{Correlated equivalent monopoles.}

With correlated equivalent monopoles, the vibrating surface is divided into squares with diagonals smaller than half the shortest acoustical wavelength of interest. ${ }^{1,4}$ Each subarea is considered as one monopole source. In Eq. (1) the source strength, $q$, of the monopoles is estimated by a measure of surface vibration, $u$, times the surface of the sub-area, $\Delta s$.

$$
q=u \cdot \Delta s
$$

\section{Piston model.}

The partial sound sources are considered to move as uncorrelated pistons and can each be modelled as a cluster of $K$ fictitious in-phase monopoles, $q_{i}$, with one equivalent monopole source strength, $q=K q_{i}$. The equivalent monopole source strength, $q$, is estimated by a measure of sound power, $P$, 